\title{
On the way to unveiling the atomic structure of superheavy elements
}

\author{
Mustapha Laatiaoui ${ }^{1,2, a}$ \\ ${ }^{1}$ Helmholtz-Institut Mainz, Staudinger Weg 18, 55128 Mainz, Germany \\ ${ }^{2}$ GSI Helmholtzzentrum für Schwerionenforschung GmbH, Planckstr. 1, 64291 Darmstadt, \\ Germany
}

\begin{abstract}
Optical spectroscopy of the transfermium elements (atomic number $Z>100$ ) is nowadays one of the most fascinating and simultaneously challenging tasks in atomic physics. On the one hand, key atomic and even nuclear ground-state properties may be obtained by studying the spectral lines of these heaviest elements. On the other hand, these elements have to be produced "online" by heavy-ion induced fusionevaporation reactions yielding rates on the order of a few atoms per second at most, which renders their optical spectroscopy extremely difficult. Only recently, a first foray of laser spectroscopy into this heaviest element region was reported. Several atomic transitions in the element nobelium $(Z=102)$ were observed and characterized, using an ultra-sensitive and highly efficient resonance ionization technique. The findings confirm the predictions and additionally provide a benchmark for theoretical modelling. The work represents an important stepping stone towards experimental studies of the atomic structure of superheavy elements.
\end{abstract}

\section{Introduction}

The experimental investigation of the atomic spectra of elements beyond fermium lies at the frontier of contemporary atomic physics research [1]. The observation of atomic transitions in these very heavy elements, for instance, would provide a stringent test of modern atomic theories and models addressing relativistic and quantum electrodynamic (QED) effects $[2,3]$. In addition, through hyperfine structure and isotope shift measurements, nuclear properties may be obtained in a comprehensive and nuclear model-independent way [4]. These properties include the spin, the magnetic dipole, and the electric quadrupole moments, and the changes in the mean-square charge radii.

However, despite the strong efforts undertaken and the large optical spectroscopy research programs initiated in the last century, optical spectroscopy was deemed to reach a barrier of applicability at the element einsteinium [5] and, since more than a decade now, at fermium [6]. The transfermium elements remained out of reach for such an approach until recently, as neither primordial isotopes of these elements exist nor can macroscopic samples be synthesized. These radioactive elements have to be produced by heavy-ion induced fusion-evaporation reactions yielding low rates, which inevitably calls for ultraefficient and fast spectroscopy techniques of extreme sensitivity. Moreover, the lack of any existing tabulated spectral lines for these elements represents a big hurdle for any laser

\footnotetext{
a e-mail: m.laatiaoui@gsi.de
}

(C) The Authors, published by EDP Sciences. This is an Open Access article distributed under the terms of the Creative Commons Attribution License 4.0 (http://creativecommons.org/licenses/by/4.0/). 
(a)

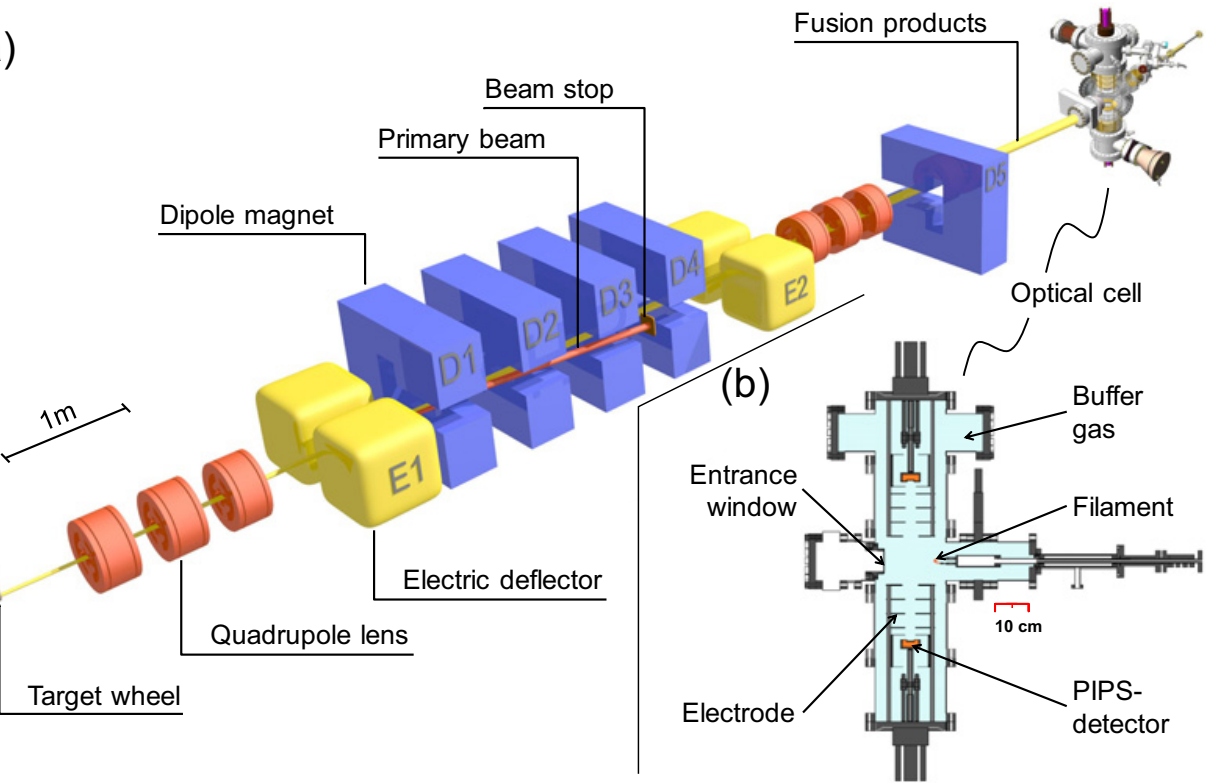

Figure 1. The experimental setup. (a) Schematic overview of the velocity filter SHIP [11] with a buffer-gas stopping cell coupled to it. A thin entrance Mylar foil separates the buffer gas volume inside the cell from the high vacuum chamber of the SHIP beamline. (b) Interior of the cell, see text for details.

spectroscopy experiment, making an extensive search for atomic transitions within a spectral range proposed by state-of-the-art atomic model calculations necessary.

Laser resonance ionization spectroscopy of the element nobelium (No) has long been anticipated at the GSI in Darmstadt [7]. To this end, the so-called RAdiation Detected Resonance Ionization Spectroscopy (RADRIS) technique was employed [8, 9]. In August 2015, atomic transitions for this element were observed for the first time, revealing atomic structure information at production rates of a few atoms per second at most [10]. Further, an upper limit value for the first ionization potential (IP) has been obtained. The investigations were focused on the radionuclide ${ }^{254} \mathrm{No}$, but were extended to even ${ }^{252} \mathrm{No}$ with the lowest production rate among the investigated isotopes.

\section{Experimental details}

Figure 1 shows the experimental setup used for laser spectroscopy of nobelium. It consists of a buffer-gas stopping cell, which was installed in the focal plane of the Separator for Heavy Ion reaction Products (SHIP) [11]. The cell exhibits an entrance window, a catcher filament, and two identical extraction electrode systems with a corresponding $\alpha$-particle detector (Passivated Implanted Planar Silicon semiconductor detector, PIPS). The radionuclide ${ }^{254}$ No with a half-life of $T_{1 / 2}=51.2 \mathrm{~s}$ was chosen for the first laser spectroscopy experiments. This was produced at SHIP in the reaction ${ }^{208} \mathrm{~Pb}\left({ }^{48} \mathrm{Ca}, 2 n\right)^{254}$ No with a cross section of about $2 \mu \mathrm{b}$ [12], cf. Table 1 . The rate of product ions delivered to the cell was regularly checked by a retractable position-sensitive 16-strip silicon detector placed in front of the entrance window.

The RADRIS measurements were organized in user-defined measurement cycles comprising a beam-on and a beam-off period to allow a temporal disentanglement of the preparation of the sample atoms from the actual spectroscopy investigations. During the 
Table 1. Nobelium isotopes investigated in the RADRIS experiments. The isotopes were produced in the $2 n$ channel of the nuclear reactions ${ }^{48} \mathrm{Ca}+{ }^{208,206} \mathrm{~Pb}$ using lead sulfide $(\mathrm{PbS})$ targets. The reaction cross sections are taken from reference [12]. The applied measurement cycles comprising beam-on and beam-off periods were optimized upon the isotope half-life [19].

\begin{tabular}{llllllll}
\hline Isotope & Half-life & $\begin{array}{l}{ }^{48} \text { Ca-beam } \\
\text { energy }\end{array}$ & $\begin{array}{l}\text { Lead } \\
\text { isotope }\end{array}$ & $\begin{array}{l}\text { PbS-Target } \\
\text { thickness }\end{array}$ & $\begin{array}{l}\text { Cross- } \\
\text { section }\end{array}$ & $\begin{array}{l}\text { Beam-on/-off } \\
\text { cycle }\end{array}$ & $\begin{array}{l}\text { RADRIS } \\
\text { efficiency }\end{array}$ \\
\hline${ }^{254} \mathrm{No}$ & $51.2 \mathrm{~s}$ & $217 \mathrm{MeV}$ & ${ }^{208} \mathrm{~Pb}$ & $470 \mu \mathrm{g} / \mathrm{cm}^{2}$ & $2 \mu \mathrm{b}$ & $25 \mathrm{~s} / 5 \mathrm{~s}$ & $(6.4 \pm 1) \%$ \\
${ }^{252} \mathrm{No}$ & $2.4 \mathrm{~s}$ & $217 \mathrm{MeV}$ & ${ }^{206} \mathrm{~Pb}$ & $460 \mu \mathrm{g} / \mathrm{cm}^{2}$ & $0.5 \mu \mathrm{b}$ & $3 \mathrm{~s} / 3 \mathrm{~s}$ & $(3.3 \pm 1) \%$ \\
\hline
\end{tabular}

beam-on period, the fusion products emerging from the target were separated from the primary beam by SHIP before entering through a $3 \mu \mathrm{m}$-thick entrance foil (Mylar) into the cell filled with 95 mbar high-purity argon gas. After thermalization, the fusion products remaining in a positive charged state were accumulated on a catcher filament, a $125 \mu \mathrm{m}$ thin tantalum wire, where they were neutralized. In a subsequent step, the fusion-product beam was turned off. Then, the accumulated atoms were evaporated from the filament, by heating this briefly to about $1350 \mathrm{~K}$. Nobelium atoms were then ionized in a two-step photoionization process [13] by pulsed lasers, see inset in Fig. 2a. In a first step $\left(\bar{v}_{1}\right)$ during this process, a valence electron is excited from the ${ }^{1} \mathrm{~S}_{0}$ ground state into a ${ }^{1} \mathrm{P}_{1}$ excited state. In a second step $\left(\bar{v}_{2}\right)$, it is excited further into the continuum beyond the ionization potential such that $\bar{v}_{1}+\bar{v}_{1} \geq$ IP leading to the ionization of the atom. Once the ionization occurred, the lasercreated ions were promptly guided by suitable electric fields to the PIPS detector where they were unambiguously identified via their unique radioactive decay fingerprint.

Up to four pulsed dye lasers operated at a pumping wavelength of $248 \mathrm{~nm}$ and one Nd:YAG pumped Optical Parametric Oscillator (OPO) operated in a frequency mixing mode were simultaneously used to search for the ${ }^{1} \mathrm{P}_{1}$ excited state in nobelium. The laser wavelengths were monitored using a HighFinesse-Ångstrom wavelength meter WS/7-UVU that was calibrated to an internal neon lamp. The second step was provided by excimer laser light at $351-355 \mathrm{~nm}$ wavelength with high power so that an efficient excitation to the continuum was possible. The reader is referred to references $[10,14]$ for more details on the method and the laser systems used in these experiments.

\section{Results}

Based on the latest multi-configuration Dirac-Fock (MCDF) $[15,16]$ and relativistic coupled cluster (RCC) [17, 18] calculations a spectral range between 28887 and $33191 \mathrm{~cm}^{-1}$ was chosen for the search for the nobelium ${ }^{1} \mathrm{~S}_{0}{ }^{1}{ }^{1} \mathrm{P}_{1}$ ground-state transition, cf. Fig. 2.

The range up to $30530 \mathrm{~cm}^{-1}$ was scanned more than twice in $\approx 0.9 \mathrm{~cm}^{-1}$ steps by the dye lasers. The OPO system scanned multiple times in $3 \mathrm{~cm}^{-1}$ steps the range between $30000 \mathrm{~cm}^{-1}$ and $33191 \mathrm{~cm}^{-1}$. The events registered during the scans were analyzed and potential candidates for the location of the resonance were identified. In the following experiments, these candidates were checked at smaller scan steps and higher statistics. In total, more than 6000 laser scan steps were conducted before discovering the resonance located at $29961.5 \mathrm{~cm}^{-1}$. Figure 2a shows a typical scan across the resonance, which is marked by an increase in the registered ${ }^{254}$ No $\alpha$-decay count rate on the PIPS detector. From the saturation characteristics for this first-step excitation, an Einstein coefficient for spontaneous emission $A_{k i}$ of $\left(4.2_{-2.8}^{+2.6}\right) \cdot 10^{8}$ /s was experimentally determined, see Fig. $2 \mathrm{~b}$. This is in good agreement with the $A_{k i}$-predictions [15-17], corroborating the observation of the ${ }^{1} \mathrm{~S}_{0^{-}}{ }^{1} \mathrm{P}_{1}$ ground-state transition. All the predicted values for the transition energy are in agreement with the measured one, which is far more precise, and thus provides a powerful benchmark. Noticeable, however, is the striking agreement of the RCC calculated value with 

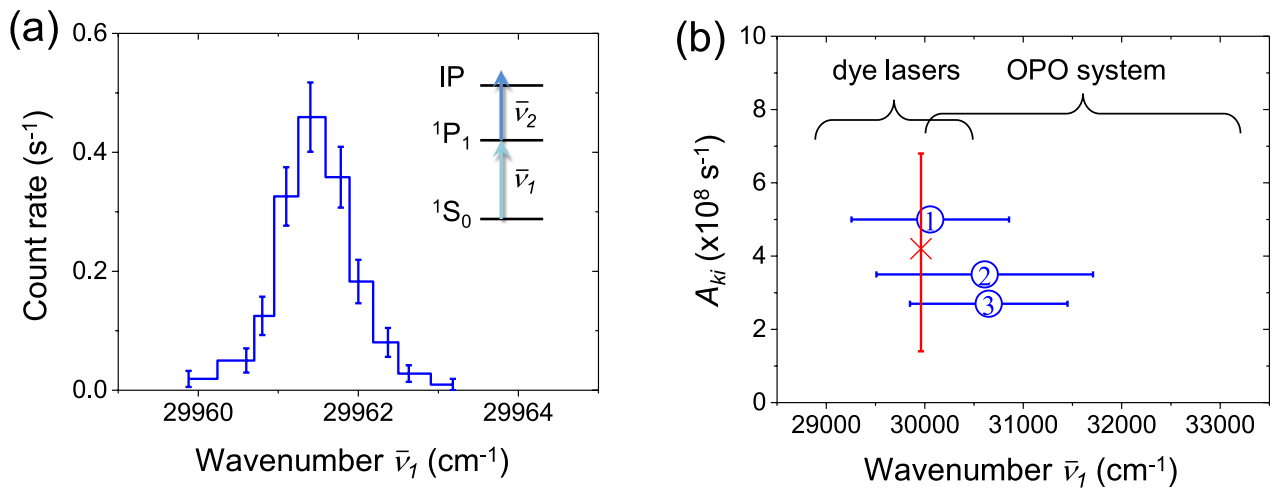

Figure 2. The first-step excitation. (a) The observed first-step resonance in nobelium in terms of registered ${ }^{254} \mathrm{No} \alpha$-decay count rates normalized to $6.2 \cdot 10^{12}{ }^{48} \mathrm{Ca}$ projectiles per second. Inset: a simplified ionization scheme. (b) Overview on the measured $(x)$ and predicted $(0)$ first-step excitation energy and the Einstein coefficient for spontaneous emission $A_{k i}$ for the ${ }^{1} \mathrm{P}_{1}$ atomic state in nobelium. The calculated values are taken from RCC- [17] (1) and MCDF calculations [16] (2) and [15] (3), respectively, with an error estimate for (2) as reported in reference [10].

the measurement. This supports that a rather conservative error estimate for the transition energy was given in reference [17]. Also worthy of mentioning is the general overestimation of the energy by all theoretical models. At least in the case of the MCDF calculations, one may attribute this to the treatment of correlation effects that positively contribute to the orbital energy.

In total, less than 10000 atoms delivered to the gas cell were sufficient to measure the shown resonance shown in Fig. 2a. The overall efficiency of the setup is determined by the ratio of the decay count rate measured with the PIPS detector at maximum of a resonance to the implantation rate of ions delivered to the cell and measured with the 16-strip silicon detector, both normalized to the primary beam intensity. This amounted to $6.4 \%$ in the case of ${ }^{254}$ No and was achieved over rather long measurement times. By conditioning the filament surface, i.e. by heating the tantalum wire under vacuum conditions to about $1800 \mathrm{~K}$, the overall efficiency was boosted for a short time by nearly a factor of two. This behaviour may be attributed to an increased retention of impurities by a clean tantalum filament surface, hence, preventing unwanted associative desorption processes. Using ${ }^{206} \mathrm{~Pb}$-targets, it was possible to extend the measurements to ${ }^{252} \mathrm{No}$ at a production cross section of only $0.5 \mu \mathrm{b}$ (cf. Table 1). It turned out that the overall efficiency strongly depends on the isotope's halflife as a result of the applied measurement cycles, which in turn were optimized with respect to the half-life. The smaller the ratio of the beam-on to the beam-off period, the smaller the efficiency. Nonetheless, the latter amounted to $3.3 \%$ for ${ }^{252} \mathrm{No}\left(T_{1 / 2}=2.4 \mathrm{~s}\right)$ proving the high efficiency of the applied spectroscopy technique.

\section{Conclusions}

Recently, the element nobelium on atom-at-a-time quantities was successfully probed by lasers, for the first time since its official discovery in 1958. At present this is the heaviest element, for which laser spectroscopic investigations were performed. The long-awaited results on the level search on nobelium confirmed a predicted strong ground-state atomic transition, the ${ }^{1} \mathrm{~S}_{0^{-}}{ }^{1} \mathrm{P}_{1}$ transition. 
From the accuracy viewpoint, the error estimates from the MCDF calculations were realistic for this transition. In comparison, RCC calculations delivered a predicted value of a striking agreement with the measured one. Given a sufficient treatment of the Coulomb correlations, the latter approach may give insights on the strength of QED effects, which could have been underestimated in nobelium so far.

In addition, the measurements revealed a high efficiency of the RADRIS setup for the different nobelium isotopes, paving the way for isotope shift and hyperfine structure studies in the region of deformed nuclei around the neutron shell closure $N=152$ and $Z=102$. Moreover, the heaviest actinide element, lawrencium, which is produced at lower rates than the investigated nuclides and exhibits a different atomic structure, is now within reach of RADRIS experiments.

The results thus provide valuable anchor points for theoretical models and opens up new perspectives for future precision measurements of atomic and nuclear properties of the elusive transfermium elements using laser spectroscopy.

\section{References}

[1] H. Backe et al., Nucl. Phys. A944, 492 (2015).

[2] P. Schwerdtfeger et al., Nucl. Phys. A944, 551 (2015).

[3] E. Eliav, S. Fritzsche, U. Kaldor, Nucl. Phys. A944, 518 (2015).

[4] P. Campbell, I.D. Moore, M.R. Pearson, Prog. Part. Nucl. Phys. 86, 127 (2016).

[5] E.F. Worden et al., in The Chemistry of the Actinide and Trans-actinide Elements, eds. L.R. Morss, M. Edelstein, J. Fuger, vol. 3, chap. 16.4 (Springer, 2008).

[6] M. Sewtz et al., Phys. Rev. Lett. 90, 163002 (2003).

[7] H. Backe et al., Eur. Phys. J. D45, 99 (2007).

[8] W. Lauth et al., Phys. Rev. Lett. 68, 1675 (1992).

[9] M. Laatiaoui et al., Eur. Phys. J. D68, 71 (2014).

[10] M. Laatiaoui et al., Nature (2016), in press. http://dx.doi.org/10.1038/ nature19345.

[11] S. Hofmann and G. Münzenberg, Rev. Mod. Phys. 72, 733 (2000).

[12] Yu.Ts. Oganessian et al., Phys. Rev. C 64, 054606 (2001).

[13] V.S. Letokhov, Laser Photoionization Spectroscopy (Academic Press, 1987).

[14] F. Lautenschläger et al., Nucl. Instr. Meth. B383, 115 (2016).

[15] P. Indelicato et al., Eur. Phys. J. D45, 155 (2007).

[16] Y. Liu, R. Hutton, Y. Zou, Phys. Rev. A 76, 062503 (2007).

[17] A. Borschevsky et al., Phys. Rev. A 75, 042514 (2007).

[18] V.A. Dzuba, M.S. Safronova, U.I. Safronova, Phys. Rev. A 90, 012504 (2014).

[19] M. Laatiaoui et al., Hyperfine Int. 227, 69 (2014). 